\title{
Updated results of the Horn Study for the Nufact
}

\author{
A. E. Ball ${ }^{a}$, A. Blondel ${ }^{b}$, \\ S. Gilardoni ${ }^{b 1}$, N. Vassilopoulos ${ }^{a}$ \\ a Cern, CH-1211 Geneve 23, Switzerland \\ ${ }^{b}$ Département de Physique, Université de Genève, Switzerland
}

\begin{abstract}
An updated study of a magnetic horn as a possible option for the pion collection system at the Neutrino Factory is presented. The pion yield is similar to that obtained from the standard benchmark solenoid.
\end{abstract}

\footnotetext{
${ }^{1}$ supported by the CERN Doctoral Student Program.
} 


\section{Introduction}

A horn focusing system for pions was proposed in [1] as a possible alternative to the standard solenoid [2] used for the front end of the Neutrino Factory. Advantages of horns are that i) they are sign selective, ii) they are radiation hard and the parts exposed to the radiation are relatively cheap, iii) there is some freedom to match the shape of the horn to the most favourable phase space accepted downstream, in the phase rotation and cooling channel. Studies presented in [1] are not directly comparable to those of the solenoid because of a different particle generation code, and because they concentrate on a thin target. Further studies are presented here, with which a comparison can be made.

\section{Target simulation}

In the CERN proton driver scheme, the energy of the incoming proton beam is $2.2 \mathrm{GeV}$. The pencil-like beam is parallel to the horn axis.

The protons impinge on a mercury target which is a cylinder $30 \mathrm{~cm}$ long $(\approx$ two interaction lengths) and $0.75 \mathrm{~cm}$ radius. We chose these dimensions to reproduce the same conditions as those studied for the pion collection with a solenoid [2]. The target is positioned inside the horn at the zero of the coordinate system, and it is parallel to the beam axis. This geometry is to be considered only for simulation purposes; we refer to [5] for a first realisation of a mercury jet. The secondary particle production is simulated by MARS [4].

\section{Simulation}

As presented in [1], the pions generated by MARS are transported through the horn magnetic field by GEANT [6]. Pions are counted at the exit of the horn and after one meter of drift space with no magnetic field. We considered different geometries for the horn inner conductor design, and different currents. The best pion capture is obtained by the triangular horn shape shown in Figure 1 and described in Table 1 with $300 \mathrm{kA}$. This current is estimated as a conservative value, taking into account mechanical and heating considerations. The simulation includes pion absorption due to the inner conductor material, which is aluminium. The thickness is about $16 \mathrm{~mm}$ in 


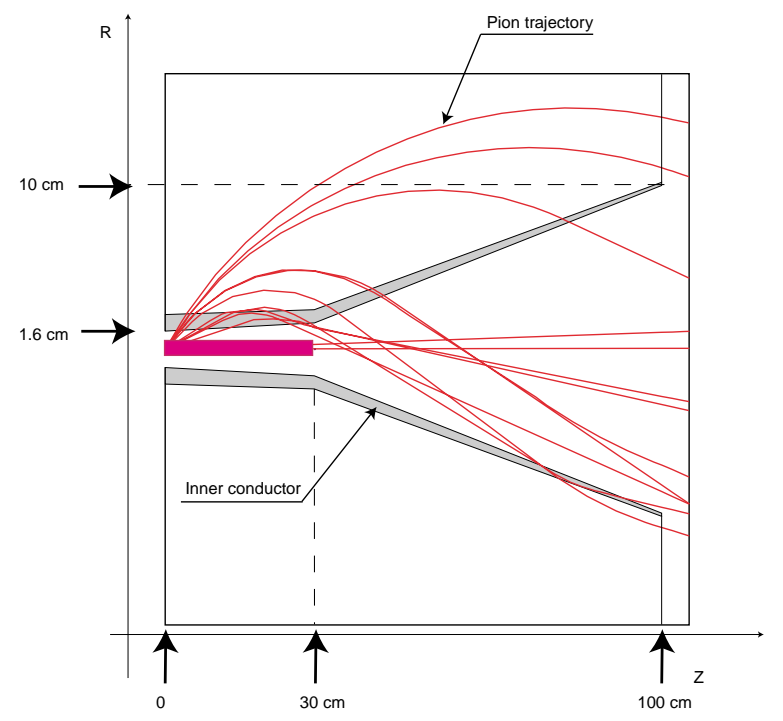

Figure 1: Pion trajectories for triangular horn shape

the section around the target and decreases to $1.8 \mathrm{~mm}$ downstream of the horn. This particular size was calculated by doubling the conductor section of the existing horn at CERN, where the current peak is $150 \mathrm{kA} \mathrm{[7].}$

\begin{tabular}{||c|c||}
\hline $\mathrm{z}(\mathrm{m})$ & $\mathrm{R}(\mathrm{cm})$ \\
\hline \hline 0.0 & 1.6 \\
\hline 0.3 & 2.0 \\
\hline 1.0 & 10.0 \\
\hline
\end{tabular}

Table 1: Inner conductor coordinates for the triangular horn.

\section{Results}

Table 2 gives the number of $\pi^{+}$per POT (Proton On Target) per $\mathrm{GeV}$ collected at the exit, and at one meter after the end of the horn, with no matching solenoid ${ }^{2}$. In the case of solenoid capture, however, the pions are

\footnotetext{
${ }^{2}$ Similar results are found when a simulation with only MARS is run for the horn.
} 
counted in a $1.25 \mathrm{~T}$ transport solenoid, one meter after the adiabatic section [2]. The $2 \mathrm{GeV}$ and the $16 \mathrm{GeV}^{3}$ yields for the benchmark solenoid are computed from [3] and are shown in Table 3. The pions are selected in space in a radius of $30 \mathrm{~cm}$ and for a momentum window defined by $0.05<\mathrm{p}<0.8$ $\mathrm{GeV} / \mathrm{c}$.

\begin{tabular}{||c|c|c||}
\hline Horn & $\pi^{+} /$POT $/ \mathrm{GeV}$ & \\
\hline \hline $300 \mathrm{kA}$ & 0.021 & no material absorption \\
after $1 \mathrm{~m}$ & 0.014 & \\
\hline $300 \mathrm{kA}$ & $\mathbf{0 . 0 1 6}$ & with material absorption \\
after $1 \mathrm{~m}$ & 0.010 & \\
\hline $400 \mathrm{kA}$ & 0.020 & with material absorption \\
after $1 \mathrm{~m}$ & 0.014 & \\
\hline
\end{tabular}

Table 2: Flux of $\pi^{+}$at the exit and 1 meter after the horn, with different currents and with and without material absorption for the inner conductor.

\begin{tabular}{||c|c|c|c||}
\hline Solenoid & MARS & MARS+GEANT321 & MARS 16 GeV \\
\hline \hline$\pi^{+} / \mathrm{POT} / \mathrm{GeV}$ & $\mathbf{0 . 0 1 7}$ & 0.020 & 0.025 \\
\hline
\end{tabular}

Table 3: Flux of $\pi^{+}$for solenoid capture after $1 \mathrm{~m}$ in the $1.25 \mathrm{~T}$ field for different simulation.

\section{Conclusions}

The performance of the triangular horn is comparable to that of the benchmark solenoid. Nevertheless we need to study and optimise the matching solenoid that connects the focusing system to the decay channel and the first section of phase rotation. A preliminary study shows that there is only a small loss of pions in the matching regions. However, a further study should quantify this loss and verify the performance of a possible reflector after the horn.

\footnotetext{
${ }^{3}$ For $16 \mathrm{GeV}$ the flux is rescaled to $1 \mathrm{GeV}$, although $\pi$ production does not scale exactly linearly with proton energy.
} 


\section{References}

[1] A. Ball et al., "Preliminary Magnetic Horn Studies in the Collection Scheme for a Neutrino Factory", proceedings of the NuFact'99 (to be published by Elsevier)

[2] N. Mokhov, "PI/MU Yield and power dissipation for carbon and mercury targets in 20-Tesla solenoid with matching section", MUCOLL NOTE 61

[3] N. Mokhov's courtesy, http://www-ap.fnal.gov/mokhov/mumu/target99/

[4] N.V. Mokhov, "The MARS Code System User's Guide", Fermilab-FN-628 (1995)

N.V. Mokhov, "MARS Code Development, Benchmarking and Applications" Fermilab-Conf-00-066(2000)

O.E. Krivosheev and N.V. Mokhov, "A New MARS and its Applications", Fermilab-Conf-98/43 (1998)

N.V. Mokhov, S.I. Striganov, A. Van Ginneken, S.G. Mashnik, A.J. Sierk and J. Ranft, "MARS Code Developments", Fermilab-Conf-98/379 (1998)

[5] C.D.Johnson, "Study of the Behaviour of a Pulsed Liquid Jet Target", Neutrino Factory Note 27

[6] R. Brun et al., GEANT321, CERN Library

[7] The CERN Neutrino Beam to Gran Sasso, CERN 98-02, INFN/AE-98/05 and Addendum, CERN-SL/99-034(DI), INFN/AE-99/05 